\title{
DOSB Informationen
}

Ger J Exerc Sport Res 2022 $52: 206-208$

https://doi.org/10.1007/s12662-022-00799-x

(c) The Author(s), under exclusive licence to Springer-Verlag GmbH Deutschland and Bundesinstitut für Sportwissenschaft, Deutscher Olympischer Sportbund, Deutsche Vereinigung für Sportwissenschaft 2022
Deutscher Olympischer Sportbund (DOSB)

Melinda Strätz

Otto-Fleck-Schneise 12

D-60528 Frankfurt am Main

straetz@dosb.de

www.dosb.de

Unter der Mitarbeit von Prof. Dr. Detlef Kuhlmann,

Institut für Sportwissenschaft der Leibniz Universität Hannover

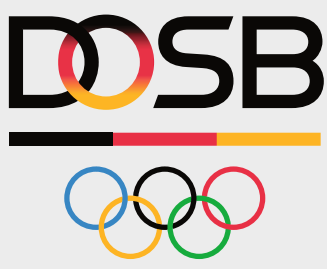

\section{Auftakttreffen des Beirats im GeniAI Projekt des DOSB}

Der Projektbeirat für das „GeniAl“-Projekt mit dem Titel "Gemeinsam bewegen - Gesund leben im Alter" (GeniAl) hat sich im November 2021 noch unter der Leitung des (jetzt ehemaligen) Vizepräsidenten für Breitensport und Sportentwicklung des Deutschen Olympischen Sportbundes (DOSB), Andreas Silbersack, im digitalen Raum konstituiert und erste Fragestellungen zu den Themenfeldern Gesundheit, Integration und Senior*innen diskutiert. Elf Expert*innen aus Wissenschaft, Politik und Praxis waren dazu eingeladen. Die Beiratsmitglieder kommen aus Bereichen und Organisationen, die eng mit den Themenfeldern des GeniAl Projektes verknüpft sind. Dieser Querschnitt ermöglicht es, optimal auf die übergeordneten Fragestellungen und Herausforderungen des Projektes einzugehen. Neben dem inhaltlichen Austausch und Input sind der Transport von Informationen und Erkenntnissen vom Projekt in die Netzwerke der Expert ${ }^{\star}$ innen Ziele des Beirates.

Diebeiden Projektleiter*innen Katja Lüke und Jakob Etzel gaben dazu eine Übersicht über die Inhalte des Gesamt- sowie der fünf Teilprojekte. Susanne Huth vom Institut INBAS-Sozialforschung, für das Projekt-Coaching und die Evaluation mit im Team, in- formierte über den Stand und die Planungen der wissenschaftlichen Evaluation. Die Bedeutung des Themas und der bestehende Bedarf an gesundheitspräventiven Maßnahmen für ältere Migrant*innen wurde von allen dabei besonders betont. Zur Herausforderung der Zugangswege zur Zielgruppe wurden verschiedene Vorschläge eingebracht, die zum Teil in den fünf verschiedenen Teilprojekten bereits umgesetzt werden. Als eine besondere Herausforderung wurden die Corona-Pandemie und die leider notwendigen Einschränkungen für Treffen im Aufbau der Projektphase gesehen. In der dreijährigen Projektlaufzeit ist damit der Aufbau von Bewegungsgruppen in einigen Projekten erschwert. Das DOSB Team von GeniAl freut sich auf den zukünftigen Austausch mit dem Beirat, der zweimal jährlich tagen wird.

$\mathrm{Zu}$ dem hochkarätig besetzen Beirat gehören: Dr. Erik Nils Voigt (Bundesministerium des Inneren, für Bau und Heimat), Dr. Sonja Stupp (Bundesministerium für Gesundheit), Andrea Schumacher (BAMF, Bundesamt für Migration und Flüchtlinge), Dr. Cornelia Geukes (BAGSO, Bundesarbeitsgemeinschaft der Seniorenorganisationen), Dr. Elke Olbermann (Forschungsgesellschaft für Gerontologie (FfG), TU Dort- mund), Stefan Bräunling (Kooperationsverbund Gesundheitliche Chancengleichheit), Dr. Natalia Roesler (bbt, Bundeselternnetzwerk der Migrantenorganisationen für Bildung \& Teilhabe), Jens Hupfeld (GKV Spitzenverband), Sieglinde Ludwig (BVPG, Bundesvereinigung Prävention und Gesundheitsförderung), Ali Toprak (BAGIV, Bundesarbeitsgemeinschaft der Immigrantenverbände) und Ramazan Salman (Ethno-Medizinisches Zentrum e.V.). Das DOSB-Projekt GeniAl wird im Rahmen des Bundes- programms „Integration durch Sport“ (IdS) vom Bundesministerium des Innern und Heimat (BMI), dem Bundesministerium für Gesundheit (BMG) und dem Bundesamt für Migration und Flüchtlinge (BAMF) gefördert. Es ist ein Kernvorhaben des $\mathrm{Na}-$ tionalen Aktionsplans Integration (NAP-I), das bis Ende 2023 umgesetzt werden soll. Weitere Informationen zum Projekt gibt es auch auf der GeniAl Website: https:// integration.dosb.de/inhalte/ projekte/gesundheitsfoerderungaelterer-migrantinnen.

\section{DOSB-Projekt zu Sport und Demenz in der Praxisphase}

Der Deutsche Olympische Sportbund (DOSB) hat vor rund einem Jahr mit Unterstützung des Bundesministeriums für Familie, Senioren, Frauen und Jugend (BMFSFJ) das Projekt „Sport bewegt Menschen mit Demenz" initiiert und arbeitet dabei sehr eng mit den Demenz-Expert*innen der Deutschen Alzheimer Gesellschaft (DAlzG) zusammen. Sport kann den Krankheitsverlauf verzögern helfen und die Lebensqualität von Menschen mit Demenz (und ihren Angehörigen) erhöhen. Das Projekt ist 2021 mit vier Mitgliedsorganisationen des DOSB in seine Praxisphase gegangen. So bieten z.B. die Tischtennisvereine VfL Fortuna Marzahn und der Steglitzer Tischtennis Klub in Berlin sowie drei weitere Tischtennisvereine in Stuttgart und Mandelsloh im Rahmen des Teilprojekts beim Deutschen Tischtennis-Bund Sport für Menschen mit Demenz und ihre Angehörigen an. Beim Landessportbund NordrheinWestfalen sind mehrere Vereine an den Start gegangen, die auch das von der Deutschen Sporthochschule Köln konzipierte „Denksport-Modell“ testen. Der Anspruch des Deutschen TurnerBundes mit zehn verschiedenen Vereinen in Frankfurt am Main ist es, beim Sport für Ältere sen- 
sibler für auftretende Demenzerkrankungen zu werden und Menschen mit Demenz möglichst (lange) in den Turn- und Sportvereinen zu halten.

Allerdings ist es gar nicht so einfach, Vereinsangebote zu initiieren, und die Gegebenheiten auf dem Land mit zum Teil nur einem Sportverein im Dorf sind andere als in der Stadt, wie die Arbeit der Sportregion Osnabrück-Stadt und Land und des Landessportbundes Niedersachsen zeigt. Erschreckend ist auch die Information, dass Menschen mit Demenz zum Teil in andere Orte fahren, um dort das Vereinsangebot wahrzunehmen, da sie sich für ihre Erkrankung schämen. Das Deutsche Zentrum für neurodegenerative Erkrankungen (DZNE) am Standort Greifswald begleitet das Projekt wissenschaftlich und hat zunächst eine Befragung gestartet, in der die Besteller der Materialbox „Sport bewegt Menschen mit Demenz", die dank der Förderung durch BMFSFJ in einer dritten Auflage produziert werden kann, befragt werden, wie sie diese einsetzen. Bei der Abschlussveranstaltung am 12. Mai 2022 wird es darum gehen, wie bundesweit noch mehr Angebote für Menschen mit Demenz entstehen können. Weitere Informationen zum Projekt „Sport bewegt Menschen mit Demenz" auch im Internet unter: richtigfitab50.dosb.de/demenz

\section{Sammelband über „Bildungspotenziale der Olympischen Idee"}

\begin{abstract}
Die neue Publikation „Bildungspotenziale der Olympischen Idee" bietet ein breites Spektrum an Perspektiven auf das Thema Olympische Spiele und damit eine Grundlage für eine fundierte Auseinandersetzung. Der gleichnamige Sammelband ist nun als frei verfügbare Open-AccessPublikation sowie als gedruckte Ausgabe erhältlich. Die Olympische Bewegung steht nicht erst seit Beginn der Covid-19-Pandemie oder den Debatten um die Wahl Pekings zum Gastgeber der Winterspiele 2022 vor vielfältigen Herausforderungen. Olympische Spiele polarisieren und bewegen sich beständig im Spannungsfeld zwischen idealisierter und unkritischer Begeisterung auf der einen und strikter, fast feindlicher Ablehnung auf der anderen Seite. Eine objektive Beschäftigung mit dem Ist-Zustand der Olympischen Bewegung ist eher die Ausnahme.
\end{abstract}

Genau an dieser Stelle setzt die neue Publikation an, die von der Sportwissenschaftlerin Prof. Dr. Stefanie Pietsch (Universität Regensburg) angestoßen und gemeinsam mit der Deutschen Olympischen Akademie (DOA) umgesetzt wurde. Der Sammelband verfolgt sowohl einen wissenschaftlichen Anspruch als auch das Ziel, über das akademische Umfeld hinaus eine breite Leserschaft anzusprechen. Das Ergebnis ist daher eine Mischung aus wissenschaftlichen Beiträgen unterschiedlicher Disziplinen, Themen mit praktischen Bezügen sowie persönlichen Einblicken von Aktiven: „Die Idee zu diesem Projekt wurde in Olympia im Austausch mit den Mitarbeiter*innen der DOA geboren. ,Bildungspotenziale der Olympischen Idee' ist für mich ein absolutes Herzensprojekt und ich bin sehr froh, dass ich es gemeinsam mit der DOA umsetzen konnte, da mir als Pädagogin der Aspekt der Bildung in Verbindung mit Sport und Bewegung besonders am Herzen liegt. Mit diesem Werk haben wir eine sehr gute Grundlage für die persönliche Auseinandersetzung mit olympischen Themenkomplexen geschaffen", so Mitherausgeberin Prof. Dr. Stefanie Pietsch.

Auch für die DOA hat der Sammelband einen hohen Stellenwert, wie die Vorsitzende Prof. Dr. Gudrun Doll-Tepper (Berlin) erklärt: „Ich freue mich sehr, dass wir gemeinsam mit Prof. Dr. Stefanie Pietsch und den zahlreichen Autor ${ }^{\star}$ innen, die sich beteiligt haben, dieses Projekt umsetzen konnten. Der
Sammelband ergänzt das Portfolio der DOA-Publikationen und Projekte hervorragend und leistet einen wichtigen Beitrag zur konstruktiv-kritischen Begleitung der Olympischen Bewegung in Deutschland." Erschienen ist der Band „Bildungspotenziale der Olympischen Idee" im Verlag Nomos; unter folgendem Link kann das Werk frei zugänglich in digitaler Form gelesen oder als gedruckte Ausgabe bestellt werden: https://www.nomos-shop. de/academia/titel/bildungspotenziale-der-olympischen-ideeid-101390/

\section{„Kooperative Sportlandschaften" für innovative Stadtraumgestaltung}

Der Bundesverband für Wohnen und Stadtentwicklung (vhw) hat in Kooperation mit dem Deutschen Olympischen Sportbund (DOSB) eine Studie zu Kooperationen und Netzwerken von Sport und Stadtentwicklung veröffentlicht. Dass Sport und Stadtentwicklung zusammen gedacht werden sollten, scheint heute ein tragfähiger gesellschaftlicher Konsens zu sein. Trotzdem kommt es immer wieder zu Klagen darüber, dass der Sport als gesellschaftliches Potenzial und urbane Ressource in der Stadtentwicklung nicht genügend Aufmerksamkeit erfährt und die Interessen des Sports nicht ausreichend in den Planungsprozessen berücksichtigt werden. Für die Akteure der Stadtentwicklung gestaltet es sich hingegen oft schwierig, die meist rein ehrenamtlich organisierten Sportvereine oder - noch schwieriger den nichtorganisierten Sport als „Akteure“ in Planungsprozesse zur Stadtentwicklung angemessen einzubinden. Diese Erkenntnis hat den vhw dazu bewogen, das Thema „Kooperative Sportlandschaften - Netzwerke und Kooperationen im Spannungsfeld zwischen Stadtentwicklung und Sport" zum Gegenstand eines Forschungsprojektes zu machen, das von dem Sportwissenschaftler Prof. Dr. Michael Barsuhn und seinem Team vom Institut für kommunale Sportentwicklungsplanung (INSPO) an der ESAB Fachhochschule für Sport und Management Potsdam (FHSMP) durchgeführt wurde.

Die Studie zeigt, dass Kooperationen von Akteuren der Stadtentwicklung und des Sports beeindruckende Auswirkungen sowohl auf stadträumliche als auch sportimmanente Transformationsprozesse haben können. Wichtige Rahmenbedingungen für das Gelingen von Kooperationsbeziehungen sind u.a. die Sportorientierung der Kommune, eine konstante $\mathrm{Be}$ teiligung der lokalen Sportakteure über den Projektverlauf mit klar formulierten Erwartungen und Zielen, eine gute intersektorale Zusammenarbeit in- 
nerhalb der Verwaltung, feste Ansprechpersonen und Netzwerk-„Kümmerer“ sowie eine klare und inspirierende Vision, was aus der Projektkooperation am Ende entstehen kann. Abschließend werden in der Studie konkrete Handlungsempfehlungen für Kommunen, Verbände und Vereine formuliert: „Die Studie hat eine Wissensbasis geschaffen, auf deren Grundlage Handlungsempfehlungen formuliert werden, wie Akteure aus dem Sport besser in Prozessen der Stadtentwicklung mitwirken können. Ich bin zuversichtlich, dass die in diesem Bericht ver- öffentlichten Ergebnisse eine besondere Aufmerksamkeit finden werden“, so Christian Siegel, Ressortleiter „Sportstätten, Umwelt und Nachhaltigkeit" des DOSB. Die Broschüre „Kooperative Sportlandschaften" kann beim Ressort „Sportstätten, Umwelt und Nachhaltigkeit" (per E-Mail über latzel@dosb.de) kostenfrei bestellt werden und steht auch als Download Verfügung: https:// www.vhw.de/fileadmin/user_ upload/08_publikationen/vhwschriftenreihe-tagungsband/ PDFs/vhw_Schriftenreihe Nr._31_Kooperative_Sportlandschaften.pdf.

\section{Aktionsprogramm „Aufholen nach Corona für Kinder und Jugendliche"}

Noch nie zuvor haben sich Kinder und Jugendliche so wenig bewegt wie in den vergangenen zwei Jahren, gleichzeitig verbringen sie mehr Zeit vor Bildschirmen als jemals zuvor. Die Coronakrise trifft Kinder und Jugendliche besonders hart. Laut einer Studie, die in Zusammenarbeit mit dem Universitätsklinikum Hamburg Eppendorf(UKE) durchgeführt wurde, stieg die Social-Media-Nutzung auf 193 Minuten pro Tag und die Zeit, in der Kinder mit Computerspielen verbracht haben auf $139 \mathrm{Mi}$ nuten an. Um solchen Entwicklungen entgegenzuwirken hat die Bundesregierung unter dem Titel „Aufholen nach Corona für Kinder und Jugendliche“ kurz „Aufholpaket" ein bundesweites Aktionsprogramm für die Jahre 2021 und 2022 beschlossen. Das Programm wurde als Reaktion auf die starken Einschränkungen, unter denen junge Menschen in den vergangenen Monaten gelitten haben, verabschiedet. Kinder und Jugendliche benötigen besondere Unterstützung, damit die Pandemie nicht lange nachwirkt
Sportvereinen und Verbänden. Mit dem dsj-Aufholpaket sollen die Strukturen der Kinder- und Jugendarbeit im Sport gestärkt werden, um insbesondere niedrigschwellige Angebote für Bewegung, Spiel und Sport für Kinder und Jugendliche in Deutschland sowie einen Neustart der Internationalen Jugendarbeit zu ermöglichen. Auch für die neue Bundesregierung spielt das Aufholpaket eine bedeutende Rolle und wird im Koa- litionsvertrag explizit erwähnt. Somit wird dieses Programm weiterhin im Fokus stehen und auch nach 2022 aller Voraussicht nach mit einem „Zukunftspaket für Bewegung, Kultur und Gesundheit" nachhaltig gefördert werden. Weitere Informationen zum Projekt sind auch abrufbar unter: www.dsj.de/aufholpaket/. Für Rückfragen stehen in der dsj Natalie Gips und Patrick Janke via E-Mail (aufholpaket@dsj.de) zur Verfügung. und alle jungen Menschen ihre Bildungsziele erreichen sowie ihre Persönlichkeit entwickeln können. Durch die Nutzung bereits bestehender Strukturen sollen Angebote geschaffen werden, die schnell bei den Kindern, Jugendlichen und Familien ankommen.

Das Bundesministerium für Familie, Senioren, Frauen und Jugend (BMFSFJ) stellt dafür rund zwei Milliarden $€$ zur Verfügung. Das aufgelegte Aktionsprogramm ist in vier Säulen aufgeteilt, dem Abbau von Lernrückständen (Schule), Maßnahmen zur Förderung der frühkindlichen Bildung (Kita), Unterstützung für Ferienfreizeiten und außerschulische Angebote (Freizeit) sowie Kinder und Jugendliche im Alltag und in der Schule begleiten und unterstützen (Alltag). Die Deutsche Sportjugend (dsj) als eigenständige Jugendorganisation im Deutschen Olympischen Sportbund partizipiert am Bundesprogramm im Bereich Freizeit und fördert Begegnungs- und Bewegungsangebote in deutschen 